\title{
Instabilidade de Turing em um modelo matemático aplicado à epidemiologia
}

\author{
Instability of Turing in a mathematical model applied to epidemiology \\ M. O. A. Silva*; F. A. S. Silva \\ Instituto Federal do Paraná, 83215-750, Paranaguá-PR, Brasil \\ *marlon_941@live.com
}

\begin{abstract}
Neste trabalho estudamos analiticamente e numericamente um sistema matemático epidemiológico desenvolvido por Berezovsky, o qual considera uma população constituída por dois grupos: suscetíveis e infectados por uma doença arbitrária. Nesse modelo também se considera a taxa de mortalidade natural, taxa de mortalidade induzida por doença e a taxa de emigração per capita de não infectados. Para compreender o comportamento dinâmico do modelo, determinamos os pontos de equilíbrio que mostram a possibilidade de dois cenários: I) uma população livre de doenças; II) o surgimento de infectados na população. Diante desse contexto, buscamos estudar a disseminação espacial de doenças, considerando uma rede bidimensional com interação de longo alcance, que não considera apenas a interação de primeiros vizinhos, em outras palavras, essa forma de acoplamento permite que cada sítio (correspondente aos grupos formados por indivíduos que constituem a população) interaja com outros elementos da rede. As interações que ocorrem na rede são simuladas numericamente através da linguagem de programação Fortran com o integrador Lsoda com condições de contorno periódicas, utilizando condições iniciais randômicas. Para analisar a disseminação espacial de doenças no modelo de Berezovsky, determinamos as condições que satisfazem a instabilidade de Turing, na qual ocorre uma transição de estabilidade na rede, que pode ocasionar o surgimento de padrões espaciais.

Palavras-chave: Sistema Matemático Epidemiológico, Instabilidade de Turing, Interação de Longo Alcance.
\end{abstract}

The work was developed analytically and in an epidemiological mathematical system developed by Berezovsky, which is considered a population constituted by two groups: susceptible and infected by an arbitrary disease. In this case, the natural mortality rate, the mortality rate and the uninfected per capita infection rate are also considered. In order to understand the probability of two scenarios: I) a disease-free population; II) the emergence of infected in the population. Thus, we seek the spatial spread of a disease, considering a two-dimensional network with long-range interaction, which does not only matter as a firstorder interaction, in other words, a form of involvement that allows individuals who constitute the population) to interact with other elements of the network. As interactions that occur in the network, they are numerically numerous for the Formal programming language with the Lsoda integrator with periodic boundary directives, using the first random information. Abstract: In order to analyze the spatial spread of a Berezovsky disease, determine the conditions that satisfy a Turing instability, which is a change of progress in the network, which may lead to the appearance of spatial patterns.

Keywords: Epidemiological Mathematical System, Turing Instability, Long-Range Interaction.

\section{INTRODUÇÃO}

Alguns dos pioneiros em Epidemiologia Matemática foram Kermack e McKendrick (1927) [2]. Eles apresentaram, em 1927, um modelo teórico que permite compreender o comportamento de epidemia da peste bubônica que ocorreu na Índia, entre os anos de 1905 e 1906 [1]. O modelo de Kermack e McKendrick (1927) [2] descreve matematicamente a evolução temporal de uma população constituída por suscetíveis à doença, infectados pela doença e os removidos, que representam os casos de cura e também os óbitos [2].

Geralmente, modelos matemáticos epidemiológicos são compostos por equações diferenciais ordinárias (EDO), as quais podem quantificar a evolução temporal da interação entre os grupos que constituem uma população. Além de considerar a dinâmica temporal, podemos também considerar a dimensão espacial [3], como as que ocorrem nas equações de reação difusão. 
No contexto da disseminação espacial de doenças em modelos epidêmicos, alguns trabalhos utilizam a Teoria de Turing, elaborada em 1952, na área da morfogênese [4]. Na proposta de Turing explica-se matematicamente que substâncias químicas que reagem entre si e se difundem, podem formar padrões espaciais [5]. Turing demonstrou que o processo ocorre via uma quebra de estabilidade conhecida como "Instabilidade de Turing". Apesar de sua teoria ser aplicada no contexto de reações químicas, a teoria de Turing pode ser aplicada a outros sistemas espacialmente distribuídos, ou seja, também pode ser usada para descrever fenômenos de disseminação epidêmica [6].

Esse artigo tem por objetivo aplicar as hipóteses de Turing em um modelo elaborado por Berezovsky (2005) [8] com acoplamento de interação de longo alcance, considerando uma população constituída apenas por duas classes: suscetíveis e infectados pela doença. Em relação ao acoplamento espacial utilizamos a interação química, neste caso a interação decai com a distância entre os elementos da rede, semelhante a uma interação do tipo Lei de Potência. Para determinar as condições de Turing serão utilizados métodos analíticos e numéricos, considerando um sistema espacialmente estendido [7] em uma rede bidimensional.

\section{A DINÂMICA DO MODELO}

O modelo elaborado por Berezovsky (2005) [8] descreve a propagação de uma doença arbitrária através de duas equações, as quais consideram que o total da população $(N)$ é formada por dois grupos: suscetíveis à doença $(S)$ e infectados pela doença $(I)$ [8].

$$
\begin{gathered}
\frac{d s}{d t} \equiv f(S, I)=v R_{d}[(S+I)(1-S-I)]-R_{0} \frac{S I}{S+I}, \\
\frac{d I}{d t} \equiv g(S, I)=\frac{S I}{S+I}-I,
\end{gathered}
$$

sendo $R_{0}$ a taxa de reprodução da doença, a qual pode indicar o aumento da doença com $R_{0}>1$, enquanto $R_{0}<1$ pode corresponder o fim da epidemia [9]; $R_{d}$ a taxa de reprodução demográfica que indica o crescimento da população com $R_{d}>1$, enquanto $R_{d}<1$ pode implicar que a população não sobreviva [3] e $v$ é um fator associado com a redução de indivíduos que inclui a taxa de mortalidade natural, mortalidade induzida pela doença e uma taxa de emigração per-capita de não infectados [8].

A fim de entender o comportamento dinâmico das Equações (1a) e (1b) na ausência da difusão, realizamos uma análise da estabilidade linear, determinando os pontos de equilíbrio e posteriormente as suas condições de estabilidade. $O$ primeiro ponto de equilíbrio $E_{0}=$ $\left(S_{0}, I_{0}\right)$, corresponde a um estado final do sistema sem infectados:

$$
E_{0}=\left(S_{0}, I_{0}\right)=\left(1-\frac{1}{R_{d}}, 0\right)
$$

Para avaliar a estabilidade linear deste ponto de equilíbrio, utilizamos a matriz jacobiana $\boldsymbol{J}_{\mathbf{0}}$, da qual são obtidas as condições de estabilidade, avaliando os elementos na matriz jacobiana no ponto de equilíbrio:

$$
\boldsymbol{J}_{\mathbf{0}}=\left(\begin{array}{ll}
\left.\frac{\partial f}{\partial S}\right|_{\left(S_{0}, I_{0}\right)} & \left.\frac{\partial f}{\partial I}\right|_{\left(S_{0}, I_{0}\right)} \\
\left.\frac{\partial g}{\partial S}\right|_{\left(S_{0}, I_{0}\right)} & \left.\frac{\partial g}{\partial I}\right|_{\left(S_{0}, I_{0}\right)}
\end{array}\right)=\left(\begin{array}{cc}
v\left(1-R_{d}\right) & v\left(2-R_{d}\right)-R_{0} \\
0 & R_{0}-1
\end{array}\right)
$$

A partir da matriz $\boldsymbol{J}_{\mathbf{0}}$, podemos analisar os sinais dos autovalores que são expressos, por sua vez, pelo determinante $\operatorname{Det}\left(\boldsymbol{J}_{0}\right)$ e o traço da matriz $\operatorname{Tr}\left(\boldsymbol{J}_{\mathbf{0}}\right)$ : 


$$
\begin{aligned}
& \operatorname{Det}\left(\boldsymbol{J}_{\mathbf{0}}\right)=v\left(R_{0}-1\right)\left(1-R_{d}\right)>0, \\
& \operatorname{Tr}\left(\boldsymbol{J}_{\mathbf{0}}\right)=v\left(1-R_{d}\right)+R_{0}-1<0,
\end{aligned}
$$

com as condições (4a) e (4a) satisfeitas, os pontos de equilíbrio $E_{0}=\left(S_{0}, I_{0}\right)$ são considerados estáveis.

Para o segundo ponto de equilíbrio $E^{*}$ temos $I \neq 0$, ou seja, surge o grupo de infectados:

$$
E^{*}=\left(S^{*}, I^{*}\right)=\left(\frac{v R_{0} R_{d}-R_{0}-v+1}{v R_{0}^{2} R_{d}},\left(R_{0}-1\right) S^{*}\right) .
$$

A matriz jacobiana avaliada no ponto de equilíbrio $E^{*}$ é expressa por:

$$
\boldsymbol{J}^{*}=\left(\begin{array}{ll}
\left.\frac{\partial f}{\partial S}\right|_{\left(S^{*}, I^{*}\right)} & \left.\frac{\partial f}{\partial I}\right|_{\left(S^{*}, I^{*}\right)} \\
\left.\frac{\partial g}{\partial S}\right|_{\left(S^{*}, I^{*}\right)} & \left.\frac{\partial g}{\partial I}\right|_{\left(S^{*}, I^{*}\right)}
\end{array}\right)=\left(\begin{array}{cc}
-\frac{R_{0}^{2}+v R_{0} R_{d}+v R_{0}-4 R_{0}-2 v+3}{R_{0}} & \frac{2 R_{0}+2 v-v R_{0} R_{d}-3}{R_{0}} \\
\frac{\left(R_{0}-1\right)^{2}}{R_{0}} & \frac{1}{R_{0}}-1
\end{array}\right)
$$

As condições de estabilidade para os pontos de equilíbrio $E^{*}=\left(S^{*}, I^{*}\right)$, são apresentadas pelas seguintes expressões:

$$
\begin{gathered}
\operatorname{Det}\left(\boldsymbol{J}^{*}\right)=\frac{\left(v R_{0} R_{d}-R_{0}+1-v\right)\left(R_{0}-1\right)}{R_{0}}>0, \\
\operatorname{Tr}\left(\boldsymbol{J}^{*}\right)=-\left(\frac{v R_{0} R_{d}-R_{0}+1-v+\left(R_{0}-1\right)\left(R_{0}-1+v\right)}{R_{0}}\right)<0,
\end{gathered}
$$

Nas próximas seções utilizaremos esses pontos e as suas respectivas condições de estabilidade no estudo do sistema espacialmente estendido, no qual além de considerar a evolução temporal, consideraremos a dimensão espacial.

\section{O ACOPLAMENTO ESPACIAL}

Para compreender a disseminação espacial de doenças no modelo de Berezovsky (2005) [8], consideramos uma rede regular bidimensional com uma interação de longo alcance baseada na interação química de Kuramoto [10], na qual a intensidade das interações decai com a distância [7]:

$$
\begin{gathered}
\dot{S}_{k, j}=f\left(S_{k, j}, I_{k, j}\right)-D_{S}\left[S_{k, j}-\beta^{-1} \sum_{r=-N^{\prime}}^{N^{\prime}} \sum_{l=-N^{\prime}}^{N^{\prime}} K_{0}(-\gamma R)\left(S_{k+r, j+l}\right)\right], \\
\dot{I}_{k, j}=g\left(S_{k, j}, I_{k, j}\right)-D_{I}\left[I_{k, j}-\beta^{-1} \sum_{r=-N^{\prime}}^{N^{\prime}} \sum_{l=-N^{\prime}}^{N^{\prime}} K_{0}(-\gamma R)\left(I_{k+r, j+l}\right)\right],
\end{gathered}
$$

sendo $D_{S}$ e $D_{I}$ as constantes de acoplamento de suscetíveis e infectados respectivamente, $k=$ $0, \ldots, N ; j=0, \ldots, N$ as posições dos sítios, $R=\sqrt{r^{2}+l^{2}}$ a distância entre os sítios, $K_{0}$ a função de Bessel que determina a forma que decai a intensidade das interações com a distância; $\gamma$ representa a localidade da interação; $\beta^{-1}=\sum_{r=-N^{\prime}}^{N^{\prime}} \sum_{l=-N^{\prime}}^{N^{\prime}} K_{0}(-\gamma R)$ é o fator de normalização e $N^{\prime}=\frac{N-1}{2}$, sendo $N$ ímpar. 
Através das Equações (8a) e (8b), podemos analisar dois casos limites. O primeiro surge quando $\gamma \rightarrow \infty$. Neste caso, é possível mostrar que o acoplamento químico se transforma em uma interação de primeiros vizinhos, conhecida como difusiva ou local, sendo essa a interação considerada por Turing em seu trabalho. Quando aplicamos o limite de $\gamma \rightarrow 0$, obtemos a interação global, também conhecida por interação de campo médio, na qual cada sítio interage com o valor médio de outros sítios [11].

\subsection{Instabilidade de Turing}

Na teoria de Turing, utilizam-se dois processos para explicar a formação de padrões em um sistema espacialmente estendido. No contexto na Morfogênese, Turing inicialmente supôs um sistema estável, no qual ocorrem apenas às reações entre as substâncias químicas, nesta etapa não é levada em consideração a dinâmica espacial, apenas a temporal. Em um segundo momento, presumiu um acoplamento difusivo entre as células que compõem a rede. Turing mostrou que a difusão pode ocasionar uma quebra de estabilidade, processo conhecido como Instabilidade de Turing [12]. Diante desse contexto, Turing demonstrou matematicamente que um sistema que está inicialmente em um estado homogêneo, pode apresentar uma transição de estabilidade com a inclusão da interação difusiva, ocasionando na formação de padrões espaciais.

Para determinar a Instabilidade de Turing para o modelo de Berezovsky (2005) [8], devemos apresentar as condições para que ocorra a quebra de estabilidade na rede acoplada. Sendo assim, inicialmente linearizamos as Equações (8a) e (8b):

$$
\begin{gathered}
\dot{S}_{k, j}=a S_{k, j}+b I_{k, j}-D_{S}\left[S_{k, j}-\beta^{-1} \sum_{r=-N^{\prime}}^{N^{\prime}} \sum_{l=-N^{\prime}}^{N^{\prime}} K_{0}(-\gamma R)\left(S_{k+r, j+l}\right)\right], \\
\dot{I}_{k, j}=c S_{k, j}+d I_{k, j}-D_{I}\left[I_{k, j}-\beta^{-1} \sum_{r=-N^{\prime}}^{N^{\prime}} \sum_{l=-N^{\prime}}^{N^{\prime}} K_{0}(-\gamma R)\left(I_{k+r, j+l}\right)\right] .
\end{gathered}
$$

Além disso, consideramos condições de contorno periódicas para a rede acoplada, na qual analisamos os modos normais, aplicando a transformada discreta de Fourier na variável espacial para obter as equações transformadas:

$$
\left\{\begin{array}{l}
\dot{\xi}_{s k, s j}=\left[a-2 D_{S} \sigma\right] \xi_{s k, s j}+b \eta_{s k, s j} \\
\dot{\eta}_{s k, s j}=c \xi_{s k, s j}+\left[d-2 D_{I} \sigma\right] \eta_{s k, s j}
\end{array}\right.
$$

sendo $\xi_{s k, s j}$ e $\eta_{s k, s j}$ as transformadas de $S_{k, j}$ e $I_{k, j}$ respectivamente. Para determinar as condições da Instabilidade de Turing, introduzimos as seguintes notações:

$$
\begin{gathered}
\sigma=\frac{1}{2}-\beta^{-1}\left[\sum_{r=1}^{N^{\prime}} K(-\gamma r) \cos \left(\frac{2 \pi s_{k} r}{N}\right)+\sum_{l=1}^{N^{\prime}} K(-\gamma l) \cos \left(\frac{2 \pi s_{j} l}{N}\right)\right. \\
\left.+2 \sum_{r=1}^{N^{\prime}} \sum_{l=1}^{N^{\prime}} K(-\gamma R) \cos \left(\frac{2 \pi s_{k} r}{N}\right) \cos \left(\frac{2 \pi s_{j} l}{N}\right)\right], \\
a_{\sigma}=a-2 D_{S} \sigma, \\
d_{\sigma}=d-2 D_{I} \sigma,
\end{gathered}
$$

sendo $\sigma$ uma função com valores no intervalo: $0 \leq \sigma \leq 1$. Através das inequações (11a), (11b) e (11c) pode-se escrever as equações transformadas: 


$$
\left\{\begin{array}{l}
\dot{\xi}_{s k, s j}=a_{\sigma} \xi_{s k, s j}+b \eta_{s k, s j}, \\
\dot{\eta}_{s k, s j}=c \xi_{s k, s j}+d_{\sigma} \eta_{s k, s j}
\end{array}\right.
$$

A partir do sistema de equações (12), podemos determinar as condições para que ocorra a instabilidade de Turing, a qual ocorre quando o ponto de equilíbrio se transforma em um ponto sela, neste caso o determinante do sistema acoplado deve ser menor que zero. Deste modo, a condição de instabilidade será:

$$
q_{\sigma}=4 D_{S} D_{I} \sigma^{2}-2 \sigma\left(a D_{I}+d D_{S}\right)+a d-b c<0,
$$

sendo $q_{\sigma}$ o determinante da matriz jacobiana do sistema acoplado. Em vista disso, resolvemos a inequação (13) para $\sigma$ :

$$
\sigma_{ \pm}=\frac{2\left(a D_{I}+d D_{S}\right) \pm \sqrt{4\left(a D_{I}+d D_{S}\right)^{2}-16(a d-b c) D_{S} D_{I}}}{8 D_{S} D_{I}} .
$$

$\sigma_{ \pm}$delimita as regiões nas quais o sistema é estável ou instável. Para obter as condições necessárias para que ocorra a Instabilidade de Turing, definimos novos parâmetros:

logo obtemos:

$$
P=\frac{a}{D_{S}}+\frac{d}{D_{I}}, \quad Q=\frac{a d-b c}{D_{S} D_{I}} .
$$

$$
\sigma_{ \pm}=\frac{1}{4}\left(P \pm \sqrt{P^{2}-4 Q}\right)
$$

Combinando a Equação (16) com intervalo $0 \leq \sigma_{-} \leq 1$, obtemos a condição de instabilidade do sistema:

$$
0 \leq P-\sqrt{P^{2}-4 Q \leq 4}
$$

Resolvendo a inequação (17) para P, temos as condições para a Instabilidade de Turing:

$$
\left\{\begin{array}{c}
Q>2 \\
P>2 \sqrt{Q} \text { se } P \leq 4 \\
P>\frac{Q}{2}+2 \quad \text { se } P \geq 4
\end{array}\right.
$$

as condições (18) serão utilizadas para a simulação numérica do sistema acoplado.

\section{SIMULAÇÃO NUMÉRICA}

A fim de realizar uma evidência numérica para o comportamento dinâmico do sistema, inicialmente simulamos computacionalmente a rede acoplada com a interação de primeiros vizinhos com $\boldsymbol{\gamma}=\mathbf{1 0}$, na qual cada sítio interage com apenas seus vizinhos mais próximos.

Para realizar a simulação numérica, utilizamos a linguagem de programação Fortran e o integrador numérico denominado Lsoda [12] com auxílio das condições que satisfazem a Instabilidade de Turing, obtidas para modelo de Berezovsky (2005) [8]. Em vista disso, resolvemos numericamente as Equações. (8a) e (8b), considerando o espaço discreto com número de sítios $N=51$, utilizando condições iniciais randômicas e condições de contorno periódicas. Desse modo, reescrevemos as inequações (18) em termos dos parâmetros das Equações (1a) e (1b): 


$$
\left\{\begin{array}{c}
R_{d}<\frac{D_{S}\left(2 R_{0}^{2}-3 R_{0}+1\right)+D_{I}\left(4 R_{0}+2 v-R_{0}^{2}-R_{0} v-3\right)-2 P^{\prime}\left(R_{0}-1\right)}{D_{I} R_{0} v} \text { se } \\
R_{d} \geq \frac{D_{S}\left(1-R_{0}-4 R_{0} D_{I}\right)+D_{I}\left(-R_{0}^{2}-v R_{0}+4 R_{0}+2 v-3\right)}{D_{I} R_{0} v} . \\
R_{d}>\frac{\left(R_{0}-1\right)^{2}+v\left(1-R_{0}\right)-2\left[D_{I}\left(-R_{0}^{2}+4 R_{0}-v R_{0}+2 v-3\right)-2 R_{0} D_{S} D_{I}+D_{S}\left(1-R_{0}\right)\right]}{R_{0} v\left(1-R_{0}-2 D_{I}\right)} \text { se } \\
R_{d} \leq \frac{D_{S}\left(1-R_{0}-4 R_{0} D_{I}\right)+D_{I}\left(-R_{0}^{2}-v R_{0}+4 R_{0}+2 v-3\right)}{D_{I} R_{0} v}
\end{array}\right.
$$

sendo $P^{\prime}=\sqrt{D_{S} R_{0}+2 D_{I}-D_{I} R_{0}-D_{I} v-D_{S}}$. Também consideramos a condição que delimita as soluções não negativas para os pontos de equilíbrio:

$$
R_{d}>\frac{R_{0}+v-1}{v R_{0}} \text {. }
$$

A Figura 1 apresenta os padrões de Turing para diferentes valores de $R_{0}$ para analisar a distribuição espacial de infectados. A intensidade da concentração de infectados no sistema é apresentada através de uma escala de cores.

(a)

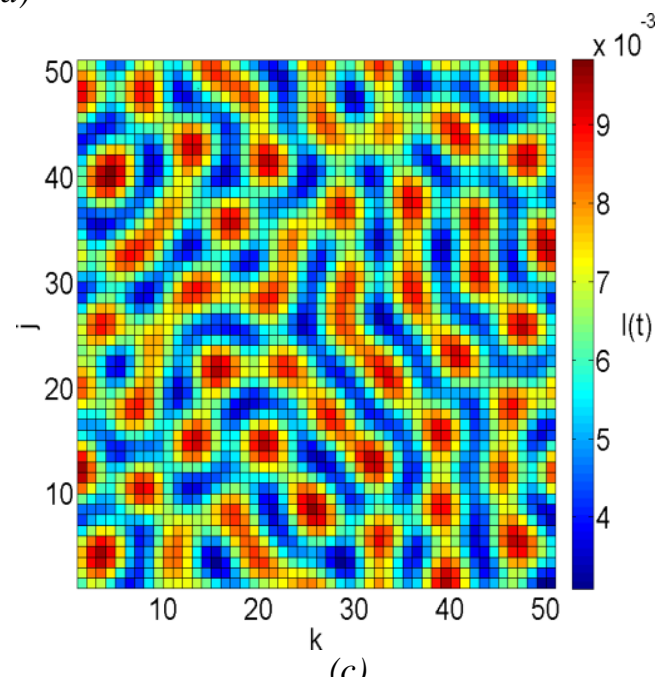

(c) (b)

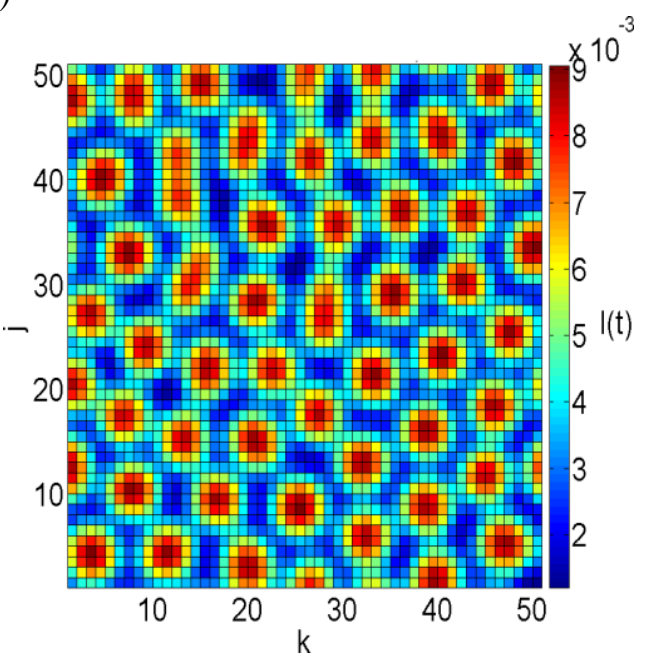

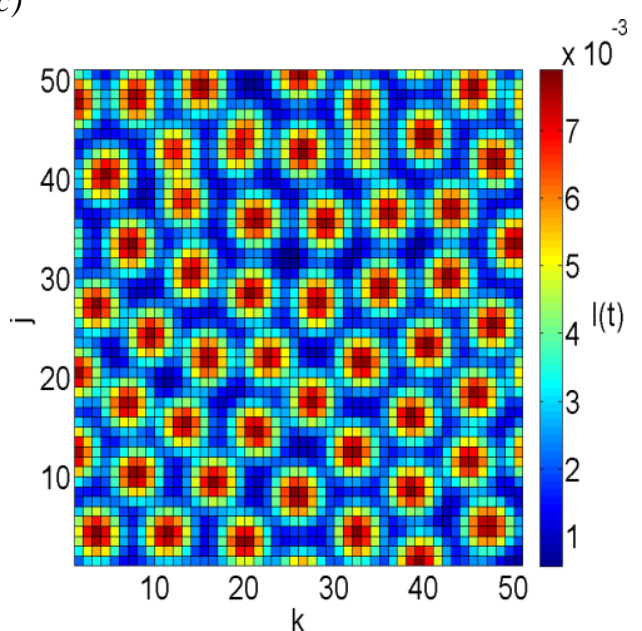

Figura 1: Padrões de Turing na distribuição de infectados no modelo de Berezovsky com parâmetros: $R_{d}=1.900, v=0.150, D_{S}=0.125$ e $D_{I}=0.550$. (a) $R_{0}=1.170$ com pontos de equilíbrio $E_{0}=$ $(0.473,0)$ e $E^{*}=(0.034,0.005),(b) R_{0}=1.180$ com pontos de equilíbrio $E_{0}=(0.473,0)$ e $E^{*}=$ $(0.015,0.002),(c) R_{0}=1.188$ com pontos de equilíbrio $E_{0}=(0.473,0)$ e $E^{*}=\left(3.351 \times 10^{-7}\right.$, $\left.6.328 \times 10^{-8}\right)$. 
Na Figura $1 a$ com $R_{0}=1.170$, verifica-se o surgimento de padrões de pontos e listras, associados à variação da densidade populacional de infectados. Nas Figuras $1 b$ e $1 c$, com $R_{0}=1.180$ e $R_{0}=1.188$ respectivamente, apresentam o surgimento de padrões relacionados a zonas isoladas de infectados com uma alta densidade relativa de infectados, enquanto as regiões remanescentes apresentam baixas proporções, as quais representam áreas que possuem poucos casos de infecções.

Diante desse contexto, verificamos que a disseminação de doenças diminui, quando $R_{0}$ está aumentando. A mesma conclusão pode ser feita na análise da série temporal do modelo na Figura 2. Nas Figuras $2 a$ e $2 b$, podemos analisar a evolução temporal dos suscetíveis e infectados, nas quais verificamos que a quantidade de infectados decai e posteriormente se estabiliza.

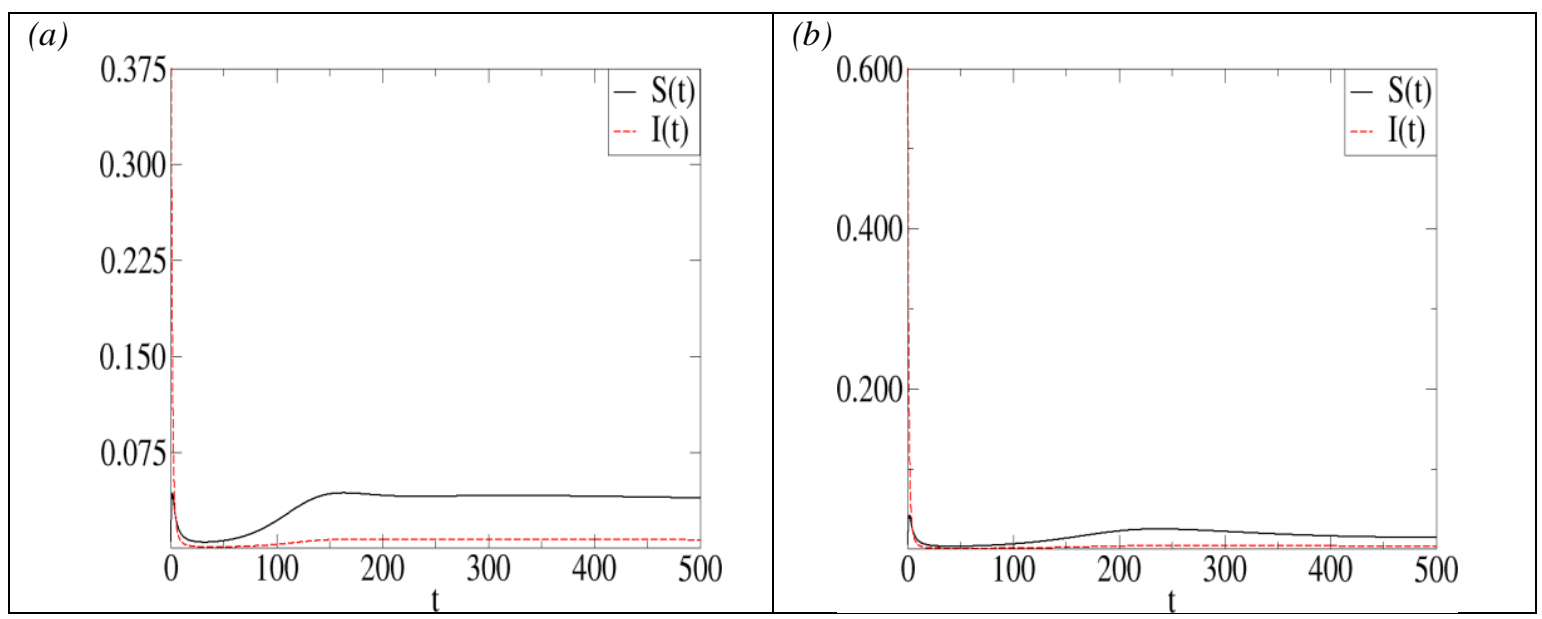

Figura 2: Série temporal de suscetíveis e infectados com os parâmetros fixos: $R_{d}=1.900, v=0.150$, $D_{S}=0.125$ e $D_{I}=0.550$, variandoo $R_{0}$. (a) $R_{0}=1.170$, $(b) R_{0}=1.180$.

Além de simular numericamente a interação de primeiros vizinhos, simulamos também um caso intermediário utilizando $\gamma=1$, no qual cada sítio interage com maior número de vizinhos. Os resultados são mostrados na Figura 3.

A Figura 3 apresenta a distribuição de infectados, considerando um caso intermediário com $\gamma=1$, no qual os elementos da rede podem interagir com uma quantidade maior de vizinhos. A Figura $3 a$ apresenta o surgimento de uma epidemia, devido ao espalhamento das doenças em grandes regiões com altos percentuais relativos de infectados. Nas Figuras $3 b$ e $3 c$, pode-se verificar zonas isoladas com alta densidade relativa da população infectada, na qual a transmissão da doença está restrita em uma determinada região, não atingindo outras áreas que podem ser consideradas seguras.
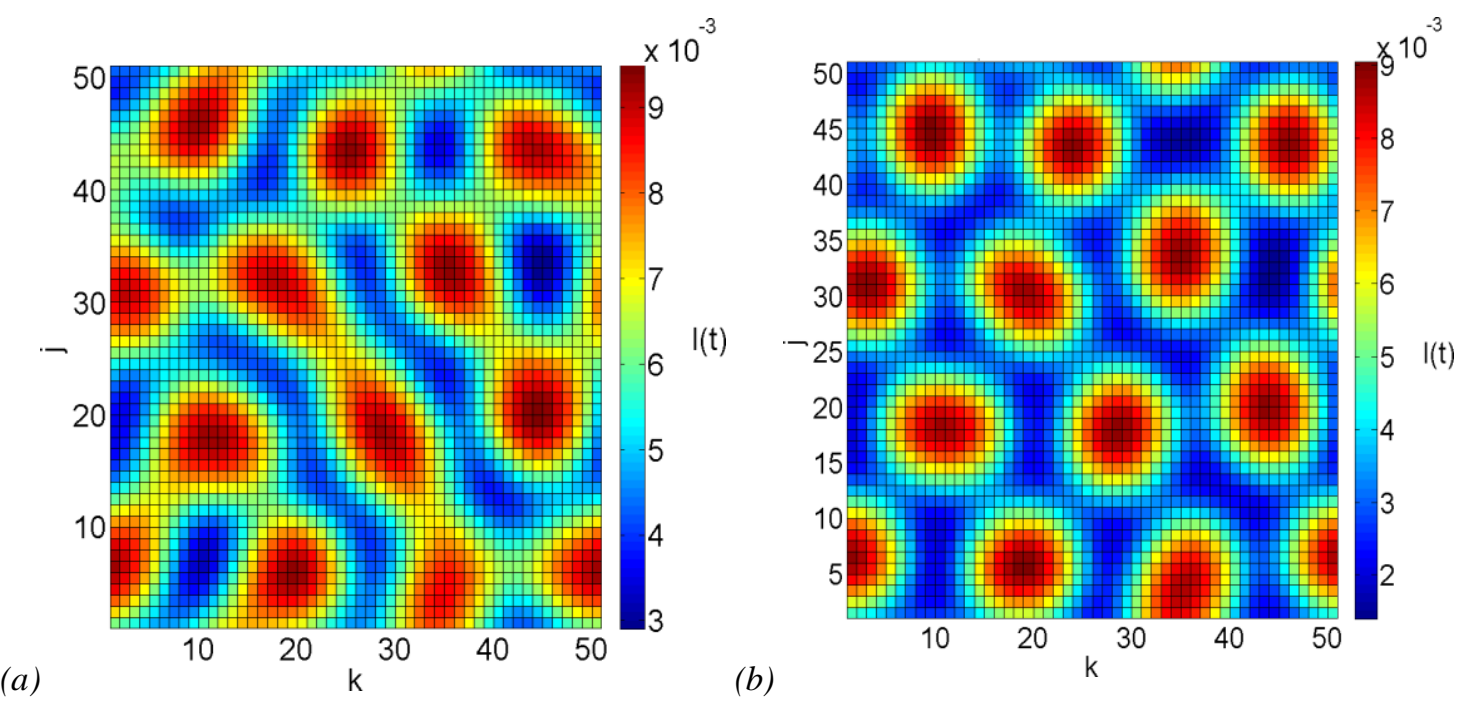


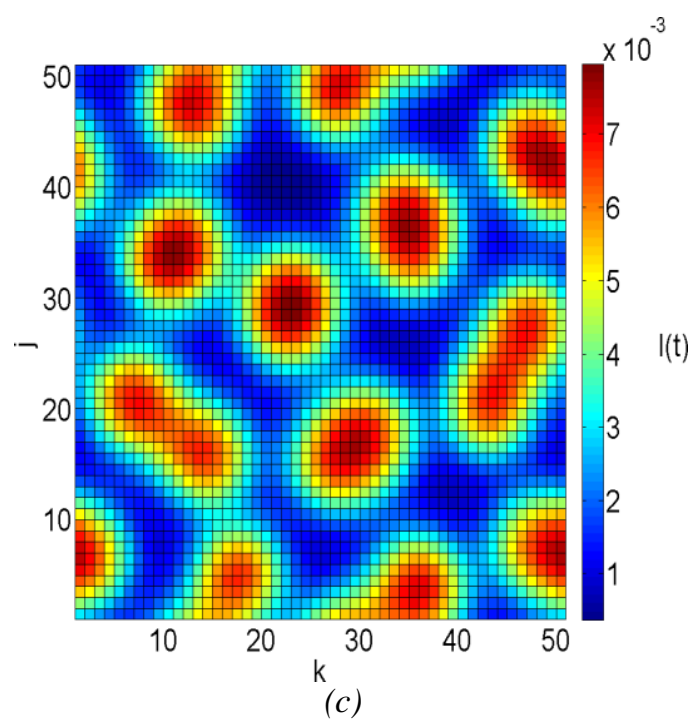

Figura 3: Padrões de Turing para $\gamma=1$, considerando os seguintes parâmetros fixos: $R_{d}=1.900$, $v=0.150, D_{S}=0.125$ e $D_{I}=0.550$, variando apenas $R_{0} .(a) R_{0}=1.170,(b) R_{0}=1.180,(c)$

$$
R_{0}=1.188
$$

\section{CONCLUSÃO}

No presente estudo, apresentamos a instabilidade de Turing em um modelo matemático aplicado à epidemiologia, considerando uma interação não local para descrever a interação entre os grupos que constituem a população. O modelo de Berezovsky (2005) [8] é descrito em apenas duas equações, as quais podem apresentar a evolução temporal de suscetíveis e infectados em uma população livre de doença ou com surgimento de doenças infecciosas transmissíveis.

A interação não local considerada é o acoplamento químico que apresenta dois casos limites: o limite $\gamma \rightarrow \infty$ que corresponde as interações entre os vizinhos próximos e o caso $\gamma \rightarrow 0$ que corresponde a uma interação global, na qual cada sítio interage com o valor médio da rede.

Para analisar a disseminação espacial de doenças, determinamos analiticamente as condições para a Instabilidade de Turing que possibilitam a formação de padrões espaciais na rede. Em seguida, simulamos numericamente uma rede com $N=51$ sítios, utilizando condições iniciais randômicas e condições de contorno periódicas.

Inicialmente consideramos a propagação de doenças com uma interação de primeiros vizinhos, com $\gamma=10$, onde fixamos todos os parâmetros e variamos apenas $R_{0}$, como foi apresentado na Figura (1). Na Figura (1a), observamos uma quantidade relativa de infectados na população, enquanto nas Figuras $(1 b)$ e (1c) verificamos o surgimento de zonas isoladas constituídas por infectados, caracterizando casos de infecções mais localizadas. Através da série temporal na Figura (2), podemos observar que após o decaimento os casos de infecções se estabilizam.

Além de analisar a disseminação de doenças com interação de primeiros vizinhos, realizamos a simulação numérica de um caso intermediário entre a interação de primeiros vizinhos e a interação global, ocasião $\gamma=1$, no qual cada sítio pode interagir com um maior número de vizinhos da rede, como mostra a Figura (3) que apresenta áreas maiores de infectados, devido a maior quantidade de interações realizadas pelos sítios. Na Figura $(3 a)$, observamos o surgimento de uma epidemia, afetando áreas maiores com altas densidades relativas de infectados na rede. Nas Figuras $(3 b)$ e $(3 c)$, podemos observar zonas isoladas, nas quais as transmissões de doenças não atingem as áreas que apresentam menor concentração da população infectada. 


\section{REFERÊNCIAS BIBLIOGRÁFICAS}

1. Monteiro L. Sistemas Dinâmicos. $3^{\text {a. }}$ ed. São Paulo: Livraria da Física; 2011.

2. Kermack W, McKendrick, A. A Contribution to the Mathematical Theory of Epidemics. Proc R Soc London A: Mathem, Phys Eng Sci. 1927;115(772):700-721, doi: 10.1098/rspa.1927.0118.

3. Wang W, et al. Turing Pattern Selection in a Reaction-Diffusion Epidemic Model. Chinese Phys B. 2011;20(7):074702, doi: 10.1088/1674-1056/20/7/074702.

4. Turing A. The Chemical Basis of Morphogenesis. Philos Trans R Soc London. Series B, Biol Sci. 1952 Ago 14;237(641):37-72, doi: 10.1098/rstb.1952.0012.

5. Lourenço C. Turing e a Morfogêneses. Bol Soc Portuguesa Matem. 2012 Ago;(67):23-59.

6. Colliza V, et al. Reaction-Diffusion Processes and Metapopulation Models in Heterogeneous Networks. Nature Phys. 2007 Mar;3:276-282, doi: 10.1038/nphys560.

7. Silva F. Formação de Padrões e Sincronização em Osciladores Acoplados [Tese], Curitiba: Universidade Federal do Paraná; 2014. 183 p.

8. Berezovsky F, et al. A Simple Epidemic Model with Surprising Dynamics. Math Biosc Eng. 2005 Jan;2(1):133-152, doi: 10.3934/mbe.2005.2.133.

9. Martcheva M. An Introduction to Mathematical Epidemiology. 1a. ed. Gainesville: Springer; 2015.

10. Silva F, Lopes S, Viana R. Synchronization of Biological Clock Cells with a Coupling Mediated by the Local Concentration of a Diffusing Substance. Comm Nonlinear Sci Num Simul. 2016;35:37-52, doi: 10.1016/j.cnsns.2015.11.003.

11. Silva F, Viana R, Prado T, Lopes S. Characterization of Spatial Patterns Produced by a Turing Instability in Coupled Dynamical Systems. Comm Nonlinear Sci Num Simul. 2014;19(4):1055-1071, doi: 10.1016/j.cnsns.2013.08.030.

12. Silva F. Formação de Padrões Espaciais em Osciladores Acoplados [Dissertação]. Curitiba: Universidade Federal do Paraná; 2010. 103 p. 